\title{
CONSIDERAÇÕES CLÍNICAS E CIRÚRGICAS SÔBRE ANEURISMAS INTRACRANIANOS
}

\author{
Mário S. Cademartori *
}

Neste trabalho consideraremos 83 casos de aneurismas intracranianos atendidos no Instituto de Neurocirurgia de Pôrto Alegre, de junho de 1960 até julho de 1967.

\section{MATERIAL E MÉTODOS}

Aneurismas de sifão carotídeo - Registramos 41 casos de aneurismas de sifão carotídeo com dados suficientes para serem computados. O paciente mais jovem (17 anos) e o mais idoso (72 anos) eram do sexo feminino (Quadro 1). Os sintomas que puderam ser relacionados com a ectasia foram: cefaléia, geralmente unilateral e retro-ocular, com duração variável entre 6 dias e 10 anos (10 casos), convulsões (4), diplopia (7), exaqueca tipo vascular (3) e paresias (5). Oito pacientes apresentavam hipertensão arterial, sendo do sexo feminino e um do sexo masculino. Os sintomas mais comuns que motivaram a hospitalização foram, por ordem de frequêencia: cefaléia de instalação súbita (33 casos), meningismo (25), paralisias ou paresia de nervos motores oculares (23), vômitos (21), ictos (9), hemiplegias ou hemiparesias (8) e convulsões (6). As combinações mais freqüentes de sintomas foram: a) cefaléias, vômitos e distúrbios visuais; $b$ ) icto, cefaléia, vômitos, distúrbios visuais, paresias ou hemiplegias; $c$ ) convulsões, cefaléia e pare-

\begin{tabular}{|c|c|c|c|c|}
\hline \multirow[t]{2}{*}{ Idade (anos) } & \multicolumn{2}{|c|}{ Sexo } & \multicolumn{2}{|c|}{ Totais } \\
\hline & M & $\mathbf{F}$ & & \\
\hline $11-20$ & & 1 & 1 & $(2 \%)$ \\
\hline $21-30$ & 3 & 4 & 7 & $(17 \%)$ \\
\hline $31-40$ & 5 & 7 & 12 & $(29 \%)$ \\
\hline $41-50$ & 1 & 12 & 13 & $(32 \%)$ \\
\hline $51-60$ & 2 & 4 & 6 & $(15 \%)$ \\
\hline $61-70$ & & 2 & 2 & $(5 \%)$ \\
\hline
\end{tabular}

* Assistente do Instituto de Neurocirurgia de Pôrto Alegre; Chefe de Clínica do Instituto de Neurocirurgia no Hospital Cristo Redentor. 
sias; $d$ ) raramente cefaléia, náuseas e ptose palpebral; e) mais raro ainda, cefaléia e ptose cerebral. O líquido cefalorraqueano era hemorrágico e hipertenso em 18, hemorrágico não tendo sido verificada a pressão em 7 , límpido em um. Os pacientes restantes não foram puncionados.

Aneurismas do grupo silviano - Registramos 19 casos (Quadro 2). Nos antecedentes e relacionados com a malformação foram registrados: cefaléia em três, perda de consciência em um, cefaléia e vômitos em um paciente. Apresentavam hipertensão arterial quatro homens e duas mulheres. Ressaltamos o fato de que os pacientes com aneurisma do sifão carotídeo foram hospitalizados, alguns vários meses e até anos depois de iniciadas as primeiras manifestações, enquanto que, entre 19 daqueles com aneurismas silvianos, 16 buscaram recursos na primeira semana. Os sintomas iniciais, em ordem de maior freqüência, foram: icto (15), meningismo (14), cefaléia (13), vômitos (7), hemiplegia (6), coma grave (5), coma vigil (4), paresias (4), convulsōes (4), agitação (3), obnubilação (2) e disartria (2 casos). Cinco pacientes apresentavam dificuldade respiratória e três foram traqueotomizados. As combinações de sintomas mais freqüentes foram: $a$ ) icto, cefaléia, vômitos, hemiplegias e afasias; $b$ ) convulsões, cefaléia, hemiplegia e afasia; c) icto, convulsões, cefaléia, hemiplegias ou paresias. o liquido cefalorraqueano era hipertenso em 12 pacientes, hemorrágico e normotenso em um, xantocrômico e com pressão normal ou pouco elevada em 4. Em um caso a reação de Wassermann resultou positiva.

\begin{tabular}{|c|c|c|c|}
\hline \multirow[t]{2}{*}{ Idade (anos) } & \multicolumn{2}{|c|}{ Sexo } & \multirow[t]{2}{*}{ Totais } \\
\hline & $\mathbf{M}$ & $\mathbf{F}$ & \\
\hline $21-30$ & & 2 & $2(10,5 \%)$ \\
\hline $31-40$ & 6 & 3 & $9(47 \%)$ \\
\hline $41-50$ & 2 & 2 & $4(21 \%)$ \\
\hline $51-60$ & 2 & & $2(10,5 \%)$ \\
\hline $61-70$ & & 1 & $1(5,5 \%)$ \\
\hline Ignorada & 1 & & 1 \\
\hline
\end{tabular}

Aneurismas do sistema cerebral anterior - Registramos 19 casos (Quadro 3). Os sintomas iniciais foram: meningismo (16), icto (9), cefaléia (5), paresias (2), convulsões (1), paraparesia e queda de temperatura ( 1 caso). As combinaçōes mais freqüentes foram a) icto, cefaléia e vômitos; b)icto e cefaléia. Quase todos os pacientes foram internados nos primeiros dias após a rotura do aneurisma. E de ressaltar a inexistência de sinais de lateralização, bem como a conservação da consciência. O líquido cefalorraqueano era hemorrágico e hipertenso em 12 pacientes. Não foram puncionados os 7 restantes.

Aneurismas do sistema vértebro-basilar - Registramos 4 casos, ou seja, $5 \%$ do total. Um paciente que apresentava síndrome meningea, paraparesia e comprometimento do terceiro nervo craniano apresentava aneurisma do tronco basilar; outro, com as mesmas manifestações, porém com hipotonia, tinha aneurisma na bifurcação basilar; em ambos foi feita angiografia dos quatro vasos e ambos fa- 


\begin{tabular}{cccc}
\hline Idade (anos) & \multicolumn{2}{c}{ Sexo } & \multicolumn{2}{c}{ Totais } \\
\cline { 2 - 3 } & $\mathrm{M}$ & $\mathrm{F}$ & \\
\hline $21-30$ & & 2 & $2(11 \%)$ \\
$31-40$ & 1 & 3 & $4(21 \%)$ \\
$41-50$ & 4 & & $4 \quad(21 \%)$ \\
$51-60$ & 3 & 4 & $7(37 \%)$ \\
$61-70$ & & 1 & $1 \quad(5 \%)$ \\
Ignorada & & 1 & 1 \\
\hline
\end{tabular}

Quadro 3 - Idade e sexo de 19 pacientes com aneurismas dos sistema cerebral anterior.

leceram. Outro paciente, com sindrome siringobulbar, apresentava aneurisma fusiforme da artéria basilar. O quarto paciente, um sexagenário, apresentou sindrome de ângulo ponto-cerebelar, havendo confirmação encefalográfica do processo expansivo; neste caso existia volumoso aneurisma da artéria basilar, cuja rotura acidental trans-operatória determinou a morte.

Aneurismas múltiplos - Registramos 6 casos $(7 \%)$ : no sifão carotídeo e na artéria silviana à direita, dois silvianos bilaterais, no ostium da artéria comunicante posterior direita, na artéria caloso-marginal do mesmo lado e início dos ramos principais da artéria silviana esquerda. Em um caso a necrópsia mostrou aneurismas na artêria silviana direita, no sifão carotídeo à esquerda e à direita, assim como na artéria cerebral posterior direita. $\mathrm{Em}$ um caso a verificação foi trans-operatória: rotura de aneurisma do sifão carotídeo à esquerda, durante intervenção cirúrgica visando a aneurisma silviano à direita.

Angiografia e localização dos aneurismas - $\mathrm{O}$ estudo angiográfico foi efetuado entre o fim da primeira e inicio da segunda semana em 36 pacientes. No cômputo geral foi feito desde o segundo até o sexagésimo dia após a primeira hemorragia. Foi unilateral em 66 casos, bilateral em 11 carótido-vertebral em 4 . Em dois casos, o aneurisma constituiu achado operatório. Imagens interpretadas como "espasmos" arteriográficos foram vistas em 11 casos de aneurismas do sifão carotídeo, em 11 da artéria silviana e 8 da artéria comunicante anterior, ou seja, respectivamente, em $27 \%$, $58 \%$ e $53 \%$ dos casos para os mesmos grupos. Os dados referentes a localização dos aneurismas constam no quadro 4 .

Ressangramentos - Presumimos ter havido ressangramento levando em consideração a reagudização da cefaléia, o reaparecimento de meningismo, o agravamento ou instalação de novos déficits neurológicos, a deterioração do estado de consciência e as manifestações neuro-vegetativas. Assim considerando, houve ressangramento em 17 pacientes $(20 \%)$, dos quais 7 apresentavam aneurismas no sifão carotídeo, 3 da artéria silviana e 7 da artéria comunicante anterior. Ocorreu ressangramento nas duas primeiras semanas em 14 casos ( 6 do sifão carotídeo, 3 da silviana e 5 da comunicante anterior); na terceira semana houve ressangramento em 3 casos (um de sifão carotídeo e dois da artéria comunicante anterior). Vieram a falecer 12 pacientes com aneurismas ( 7 da comunicante anterior, 3 do sifão carotideo e dois da artéria silviana), ou seja, $70 \%$ dos pacientes nos quais ocorreu ressangramento. 
A. carótica interna (41 casos, $49 \%$ )

no sifão
intracavernoso
região da a. oftálmica
região da a. comunicante posterior
comunicante posterior à bifurcação
bifurcação

$\begin{aligned} 1 & (1,2 \%) \\ 1 & (1,2 \%) \\ 1 & (1,2 \%) \\ 29 & (34,6 \%) \\ 5 & (6,0 \%) \\ 4 & (4,8 \%)\end{aligned}$

A. silviana (19 casos, $23 \%$ )

porção inicial aos ramos principais ramos principais

A. cerebral anterior (19 casos, $23 \%$ )

porção inicial

$1(1,2 \%)$

comunicante anterior

$15(18,0 \%)$

pericalosa

$3(3,8 \%)$

A. basilar ( 4 casos, $5 \%$ )

tronco

$3(3,8 \%)$

bifurcação

$1(1,2 \%)$

Quadro 4 - Localização dos aneurismas em 83 casos.

\section{TRATAMENTO CIRÚRGICO}

Tratamento cirúrgico direto dos aneurismas do sifão carotídeo - Foram operados 27 pacientes, 3 em más condições (grupo IV) ${ }^{4}$ e 24 em situação clínica satisfatória (grupos I e II)*. O menor período entre o internamento e a intervenção cirúrgica foi de 10 dias e, o mais longo, de 90 dias. A maioria dos pacientes foi submetida à intervenção a partir do $12 .^{\circ}$ dia, não constam dados a êste respeito em 5 casos. Todos os pacientes foram operados em posição sentada e mediante abordagem têmporo-frontal. Como recursos auxiliares foram usados: drenagem espinhal contínua (12), drenagem espinhal associada a administração de uréia (2), uréia exclusivamente (2), hipotensão arterial e lobectomia temporal (2), hipotensão e drenagem espinhal (1), punção ventricular (1 caso). Não foi usado qualquer recurso auxiliar em cinco casos; não constam dados a êste respeito em dois casos. Os aneurismas foram clipados (14), envolvidos com músculo martelado (3) e com gelfoam ( 1 caso); não consta o procedimento em um caso. Em 8 casos houve rotura trans-operatória do aneurisma, sendo necessário clípar a artéria corótida definitivamente (4) ou temporàriamente ( 1 caso). Três pacientes em más condições pré-operatórias faleceram nas primeiras 24 horas. Dois outros faleceram no quarto dia, um sem causa aparente e outro porque o aneurisma sangrara antes da abertura da dura-mater. No oitavo dia, por encefalopatia hipertensiva, faleceu paciente no qual quatro anos antes fôra feita ligadura de carótida; houve ressangramento e o aneurisma foi excluído por clipagem. Em uma paciente, por ter escapado o clipe de Mayfield, houve ressangramento, ocorrendo o óbito no $15 .^{\circ}$ dia. No 20.0 dia faleceu um paciente com meningite e, no $5 .^{\circ}$ mês, outro no qual se desenvolveu hidrocefalia. Faleceram 10 pacientes $(37 \%)$ e receberam alta 17 , dois dos quais totalmente inválidos. Foram reoperados dois: um com hematoma extradural e outro com edema. O contrôle arteriográfico foi feito em 9 casos. 
Ligadura de carótida - Sete pacientes foram tratados mediante ligadura da carótida primitiva. Este método é utilizado levando em consideração o volume da ectasia, idade do paciente, presença de moléstia grave associada, resposta satisfatória à prova de Mattas, estado de funcionamento da artéria comunicante anterior e, principalmente, as convicções de cada cirurgião sôbre a melhor conduta em cada caso particular. Com exceção de uma, as oclusões foram executadas em só tempo. Os pacientes estavam em boas condições clínicas e dois haviam sofrido rotura do aneurisma. Em 4 dos casos a intervenção foi realizada no 5.0, $18 . .^{\circ}$,

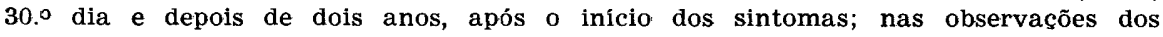
demais não constam dados a êsse respeito. Em uma paciente houve ressangramento após quatro anos e meio; o angiograma mostrou a presença do aneurisma pelo qual a carótida fôra ligada. Um paciente faleceu sùbitamente 30 dias após a ligadura. Em outro ocorreram quadro demencial e hidrocefalia, iniciados 75 dias após a oclusão da carótida. Em um caso houve necessidade de desfazer a ligadura 72 horas depois devido a instalação de hemiplegia. Os dois pacientes restantes estão bem; em um dêles a oclusão foi feita progressivamente durante 5 dias. Este método está sendo considerado com maior atenção em nosso serviço, sendo atualmente utilizado com maior freqüência.

Aneurismas da artéria silviana - Foram operados, mediante intervenção direta, 14 pacientes, dos quais cinco em más condições (grupo III - IV) e nove em boas condições clínicas. Quatro foram operados na primeira semana e os demais no início ou meados da terceira semana. A presença de hematomas é freqüente nos aneurismas da artéria silviana e, quando encontrados, facilitam a abordagem da ectasia. Em 8 casos os aneurismas foram excluídos com clipes, em 4 foram colmatados com tela de nylon, em dois com músculo martelado e gelfoam. A abordagem foi têmporo-frontal. Drenagem espinhal contínua foi utilizada em 5 e uréia em um caso ; nos demais não foi empregado qualquer método auxiliar. Em um dos casos, com aneurismas múltiplos, o aneurisma da artéria silviana foi revestido com plástico de Selverstone ${ }^{1}$. Registramos quatro óbitos nas primeiras 24 horas: em um caso, com aneurisma silviano bilateral, foi abordado o que não sangrara; em outro no final do ato cirúrgico, ocorreu hemorragia em aneurisma ignorado do sifão carotídeo do lado oposto, sendo a carótida clipada; no terceiro a necrópsia mostrou a presença de aneurismas múltiplos e cisticercose cerebral, na última houve rotura durante a disecação do aneurisma, sendo clipada a artéria silviana. A mortalidade operatória foi de $28,5 \%$. Receberam alta 10 pacientes, sendo $7 \mathrm{em}$ boas condições e dois inválidos; o estado do último paciente por ocasião da alta hospitalar não consta da observação. Contrôles pós-operatórios mediante arteriografia foram feitos em 5 pacientes: em dois foi possivel verificar estar defeituosa a colocação de clipes; em três, nos quais fôra feito revestimento com tela de nylon, observou-se exclusão do aneurisma, respectivamente 24 dias, 120 dias e três anos depois do ato cirúrgico.

Aneurismas do sistema da artéria cerebral anterior - Foram operados seis pacientes com aneurismas na artéria comunicante anterior: três em más (grupo IV) e três em boas condiçóes (grupo I) ${ }^{4}$. 0 intervalo médio entre a hemorragia e a operação foi de 20 dias. A via de acesso foi bi-frontal em três e frontal direita em três casos, num dos quais foi feita lobectomia. Dois pacientes foram operados com circulação extra-corpórea e hipotermia: em ambos houve parada cardíaca depois da queda da temperatura para $29^{\circ} \mathrm{C}$; em um dêles, com carótida trifurcada, esta foi clampeada por 10 minutos e, no outro, ambas as carótidas foram interrompidas por oito minutos. Faleceram, no 14.0 dia, dois pacientes cujos aneurismas haviam sido colmatados com músculo e gelfoam; no sexto dia, outro tratado pelo mesmo procedimento. No terceiro dia faleceu um dos pacientes que fôra operado com circulação extracorpórea com tórax aberto; o aneurisma fôra extirpado; a necrópsia nāo mostrou alteração macroscópica. Sobreviveram dois pacientes. O contrôle angiográfico mostrou, em um dêles, interrupção de ambas as artérias ce- 
rebrais anteriores e revascularização pelas anastomoses corticais; o paciente, embora sem déficits motores, está inválido. O outro sobrevivente foi operado com circulação extra-corpórea e tórax fechado; o aneurisma, em carótida trifurcada, foi colmatado com tela de nylon e, revisto dois anos depois, se apresentava com volume bem reduzido. Também foram feitas intervenções visando a aneurismas de outras porções da artéria cerebral anterior: na porção inicial em um caso, incluído no grupo IV, sendo o aneurisma clipado; o paciente sobreviveu mas ficou com distúrbios psíquicos. Em outro caso, com pequeno aneurisma na artéria pericalosa, a ectasia foi colmatada com gelfoam e a paciente teve alta em boas condições.

Não foram operados 26 pacientes, dos quais 12 por terem falecido depois de ter havido ressangramento. Os restantes não foram operados porque não aceitaram a indicação cirúrgica ou porque a intervenção foi contraindicada. Merecem destaque, neste grupo de pacientes não operados, um caso de aneurisma de sifão carotídeo com segundo sangramento, tendo o paciente tido alta, válido, nove anos depois do primeiro acidente hemorrágico e um caso de aneurisma da candelábria, considerado como de natureza micótica e que excluiu-se espontâneamente, conforme arteriografia realizada quatro meses depois da primeira hemorragia.

\section{COMENTARIOS}

Nossa experiência é pequena para que se possa tirar conclusões definitivas sôbre a melhor orientação a seguir nos casos de aneurismas intracranianos. De maneira geral optamos pela conduta cirúrgica, procurando operar pacientes em boas condições.

Já Walker 5 em 1957 dizia: "O procedimento cirúrgico adequado - ligadura da carótida no pescoço ou alguma forma de ataque intracraniano direto sôbre o aneurisma - não foi ainda estabelecido. Cada cirurgião tem a sua conduta, alicerçada na sua experiência e coragem". "Tanto neurologistas como neurocirurgiões estão começando a entender os múltiplos fatôres que necessitam ser controlados de maneira a avaliarem cientìficamente os méritos relativos à terapêutica médica e ao tratamento cirúrgico das hemorragias sub-aracnoidéias e aneurismas intracranianos, de tal maneira que, num futuro não muito distante, a controvérsia sôbre o tratamento da hemorragia sub-aracnoidéia possa ser resolvida".

As previsões de Walker começam a se concretizar. Em abril de 1966 (Neurosurgery, Vol. 16, n..$^{\circ}$ ) começaram a ser publicados os resultados do Estudo Cooperativo Americano sôbre Aneurismas Intracranianos e Hemorragias $\$$ ub-aracnodéias, de consulta obrigatória para quem se interesse pelo assunto. Entre outros, Nishioka ${ }^{2,3}$ mostrou que, com tratamento conservador, a mortalidade varia entre 36 e 100\%, pela ligadura da carótida entre 19 e $36 \%$ e, pela abordagem direta, entre 32 e $38 \%$ (Skutety e Nishioka ${ }^{4}$.

Acreditamos que o perfeito equacionamento dos múltiplos e complexos fatôres implicados no tratamento de aneurismas ainda esteja distante e, conforme preconiza Walker, o problema deve ser amplamente meditado. Acreditamos também que, com a melhora do instrumental, com o adestramento manual do pessoal especializado e com o conhecimento da conveniência e oportunidade das intervenções cirúrgicas, possam advir menores índices de mortalidade e morbilidade. 
São revistos os principais sintomas apresentados por 83 pacientes com aneurismas intracranianos nas seguintes localizações: 41 no sistema carotídeo, 19 no da artéria silviana, 19 no da artéria cerebral anterior e 4 no da artéria basilar. Como método de tratamento, a cirurgia intracraniana foi realizada em 49 pacientes, dos quais 27 apresentavam aneurisma no sifão carotídeo, 14 na cerebral média e 8 na cerebral anterior. A mortalidade operatória foi de $37 \%$ entre os pacientes com aneurisma do sifão carotídeo, de $28,5 \%$ nos do grupo da artéria silviana e de $50 \%$ nos do grupo da artéria cerebral anterior. Não houve óbito nos 7 pacientes nos quais foi feita oclusão da carótida. A morbilidade foi de $12 \%$ nos casos de aneurismas carotídeos, de $20 \%$ nos de aneurismas da cerebral média, de $50 \%$ nos de cerebral anterior e de $29 \%$ naqueles em que a carótida primitiva foi ligada. Como métodos auxiliares, foi utilizada a hipotermia com circulação extra-corpórea em dois pacientes com aneurismas da artéria comunicante anterior e drenagem espinhal contínua em 19 casos. Contrôles arteriográficos pósoperatórios, feitos em 16 casos, permitiram verificar a eficácia dos procedimentos utilizados bem como algumas falhas de técnica cirúrgica.

\section{S U M M A R Y}

\section{Clinical and surgical considerations on intracranial aneurysms.}

The clinical manifestations presented by 83 patients with intracranial aneurysms are reviewed. The site of the aneurysms were: 41 in the internal carotid, 19 in the middle cerebral, 19 in the anterior cerebral and 4 in the basilar artery. Rebleeding occurred in 17 cases; 12 of these patients died, Intracranial surgery was undertaken in 27 cases of aneuysms of the carotid, 14 the middle cerebral and 8 of the anterior cerebral artery. Seven patients were treated by occlusion of the internal carotid in the neck. Surgical mortality was of $37 \%$ among the cases of aneurysms of the carotid, of $28,5 \%$ among the middle cerebral artery cases and of $50 \%$ in those with aneurysms in the anterior cerebral artery system. There was no postoperatory death in the patients whose carotid arteries were occluded. Morbility was of $12 \%$ among the cases with aneurysms of the carotid, of $20 \%$ in the middle cerebral and of $50 \%$ in the anterior cerebral artery cases. Continuous spinal drainage during surgery with the patients in sitting positions was undertaken in 19 patients with good results. In 16 patients post-operative angiograms were performed.

\section{REFER E N CIAS}

1. SELVERSTONE, B. \& RONIS, N. - Coating and reinforcement of intracranial aneurysms with synthetic resin. Bull. Tufts New Engl. Med. Cent. 4:8, 1958.

2, NISHIOKA, H. - Evaluation of the conservative management of the ruptured intracranial aneurysms. J. Neurosurg. 25:574, 1966. 
226 ARQ. NEURO-PSIQUIAT. (SÃO PAULO) VOL. 27, N.॰ 3, SETEMBRO, 1969

3. NISHIOKA, H. - Results of the treatment of intracranial aneurysms by occlusion of the carotid artery in the neck. J. Neurosurg. 25:660, 1966.

4. SKULTETY, F. M. \& NISHIOKA, H. - The results of intracranial surgery in the treatment of aneurysms. J. Neurosurg. 25:683, 1966.

5. WALKER, A. E. - Subarachnoid haemorrhage and intracranial aneurysms: medical or surgical? Surg. 41:509, 1957.

Rua Silva Jardim 650 - Pôrto Alegre, Rio Grande do Sul - Brasil. 